\title{
Altered Expression Levels of CD59, but Not CD55, on Red Blood Cells in Stored Blood
}

\author{
Lama Al-Faris Salah Al-Humood \\ Department of Pathology, Faculty of Medicine, Kuwait University, Safat, Kuwait
}

\section{Significance of the Study}

- This study investigated the expression of CD55 and CD59 on red blood cells (RBCs) in stored blood. There is a preferential altered expression of CD59 on stored RBCs. The study adds to our understanding of storage-related progressive structural and functional changes in $\mathrm{RBC}$ that might reduce the function of and viability in RBC after transfusion.

\section{Keywords}

CD55 - CD59 $\cdot$ Stored blood

\section{Abstract}

Objective: Red blood cells (RBCs) in storage undergo structural and biochemical changes that may cause functional effects. Studies exploring structural changes affecting the expression levels of CD55 and CD59 on RBCs are limited. The aim of this study was to investigate the pattern of CD55 and CD59 expression on RBCs in stored blood from Arab donors. Materials and Methods: Flow-cytometric analysis was performed on RBCs from 92 packed RBC (PRBC) units, stored for varying times, and from 56 nonstored RBC from healthy controls using the commercial REDQUANT kit. Results: The proportions of CD55- and CD59-deficient RBCs from stored PRBC units did not significantly differ when compared with those from healthy controls; however, the mean fluorescent intensity (MFI) of CD59 expression, but not MFI of CD55 expression, on RBCs from stored PRBC units was significantly reduced when compared to the expression of RBCs from healthy controls ( $p=0.02$ ). MFI of CD55 expression on RBCs from PRBC units did not significantly differ among the 3 groups of stored RBC; however, there was a statistically significant time-dependent preferential decline in MFI of CD59 expression on RBCs from stored PRBC units $(p<0.01)$. Conclusion: There is a preferential time-dependent decline in the expression of CD59, but not of CD55, on stored RBCs, the in vivo significance of which in relation to the response to PRBC transfusion needs further investigation.

$$
\begin{aligned}
& \text { (c) } 2019 \text { The Author(s) } \\
& \text { Published by S. Karger AG, Basel }
\end{aligned}
$$

\section{Introduction}

Packed red blood cells (PRBCs) are the most commonly transfused blood component and can be lifesaving in all age groups. It has become a daily routine to use blood transfusion for treatment of patients, and the storage of

\begin{tabular}{ll}
\hline KARGER & $\begin{array}{l}\text { (อ) } 2019 \text { The Author(s) } \\
\text { Published by S. Karger AG, Basel }\end{array}$ \\
$\begin{array}{l}\text { E-Marger } \\
\text { Open access }\end{array}$ \\
www.karger.com/mpp & $\begin{array}{l}\text { This is an Open Access article licensed under the Creative Commons } \\
\text { Attribution-NonCommercial-4.0 International License (CC BY-NC) } \\
\text { (http://www.karger.com/Services/OpenAccessLicense), applicable to } \\
\text { the online version of the article only. Usage and distribution for com- } \\
\text { mercial purposes requires written permission. }\end{array}$
\end{tabular}

Salah Al-Humood

Department of Pathology, Faculty of Medicine

Kuwait University, PO Box 24923

Safat 13110 (Kuwait)

E-Mail salhumood@hsc.edu.kw 
RBCs has made this possible. Current blood banking guidelines permit the storage of human RBCs for up to 42 days before clinical use [1]. This practice is based on empirical data indicating that RBCs retain sufficient integrity for transfusion efficacy during this period (defined by the Food and Drug Administration as $75 \%$ recovery $24 \mathrm{~h}$ after transfusion) [2,3]. Twenty-four hours after transfusion, RBC survival was intended as a biological marker of corpuscular integrity in a manner analogous to increases in hemoglobin rather than as a measure of clinical benefit [4].

During ex vivo storage, RBCs intended for transfusion undergo progressive changes affecting their survival and function. They undergo metabolic and structural changes during storage, collectively referred to as the "storage lesion." Principal metabolic changes over time include declining $\mathrm{pH}$, reduced concentrations of ATP (adenosine 5 'triphosphate) and 2,3-DPG (diphosphoglycerate), and accumulation of extracellular potassium. Structural changes include membrane loss and the associated changes in RBC shape and rheology [5].

Once administered, RBCs circulate continuously in intimate contact with complement proteins, and one factor that could influence their posttransfusion survival is interaction with autologous complement in the recipient. Complement-mediated RBC lysis is normally prevented by the action of surface complement regulatory proteins, including decay-accelerating factor (DAF, CD55) and membrane inhibitor of reactive lysis (MIRL, CD59) $[6,7]$. They are glycosyl-phosphatidyl inositols (GPIs) anchored to the RBC cell surface [8]. Their regulatory function is mediated through the inhibition of $\mathrm{C} 3$ convertase formation by $\mathrm{CD} 55$, and the prevention of terminal polymerization of the membrane attack complex by CD59 [9]. CD55 and CD59 deficiencies have been considered to be responsible for complement-mediated lysis of affected RBCs from patients with the acquired condition paroxysmal nocturnal hemoglobinuria $[10,11]$; however, it has been shown that CD55 is not necessary for normal resistance to complement-mediated lysis, as individuals whose RBCs do not express CD55 (Inab phenotype) do not have abnormal sensitivity to complement [12].

Long et al. [13] have shown that surface levels of CD55 and CD59 on RBCs decline gradually during 42 days of storage; however, studies linking the efficacy of RBC transfusion to storage duration have not considered the expression levels of CD55 and CD59 as a variable that may modulate the effect of transfusion. The objective of this study was to ascertain whether the expression of CD55 and CD59 is altered on stored RBCs from Arab donors.

\section{Materials and Methods}

\section{Sample Collection}

Blood samples from PRBC units from Arab donors stored in the blood bank at the Amiri Hospital were obtained from segments attached to each unit from donors who were not known to have hematologic abnormalities; they met all routine requirements for blood donation. Blood was leukodepleted and stored at $4{ }^{\circ} \mathrm{C}$ in SAGM (saline-adenine-glucose-mannitol)-containing units according to the standard method [1]. Blood samples were obtained from PRBC units divided into 3 groups according to the following storage time frames: group 1, within the first 2 weeks of blood collection from donors; group 2, within the second 2 weeks of blood collection from donors; and group 3 , within the third 2 weeks of blood collection from donors (i.e., within 2 weeks before the expiry date of the unit). Fresh, nonstored blood samples from 56 age- and sex-matched Arab healthy controls were obtained, processed, and analyzed for the expression of CD55 and CD59 within $8 \mathrm{~h}$ of collection.

\section{Flow-Cytometric Analysis of CD55 and CD59 on RBCs}

Blood samples were collected from segments attached to the PRBC units and processed on the day of collection. An indirect immunofluorescence method was performed as described in the package insert of the REDQUANT CD55/CD59 kit (Biocytex, Marseille, France). The kit contains monoclonal antibodies to CD55 (clone 29D9) and CD59 (clone 2.24), fluorescein isothiocyanate conjugated goat anti-mouse Ig (FITC reagent), precalibrated $\mu$-beads for CD55 analysis and $\mu$-beads for CD59 analysis on RBCs, and specific buffer. Briefly, $20 \mu \mathrm{L}$ of diluted blood sample (1:150) were incubated with $20 \mu \mathrm{L}$ of antibodies to CD55 or CD59 for $10 \mathrm{~min}$ at room temperature. Two additional test tubes were added to each series, containing $40 \mu \mathrm{L}$ of precalibrated $\mu$-beads, respectively. Test tubes containing stained whole blood and beads were incubated with $20 \mu \mathrm{L}$ FITC reagent for additional $10 \mathrm{~min}$. After the staining procedure, samples were diluted with buffer $(2 \mathrm{~mL})$ and stored at $4^{\circ} \mathrm{C}$ for up to $4 \mathrm{~h}$ until measurement.

RBCs were evaluated for CD55 and CD59 expression using the REDQUANT kits according to manufacturer's instructions with the Cytomics FC 500 flow cytometer (Beckman Coulter, Inc., Brea, CA, USA). RBCs were gated on the basis of their forward and side scatter characteristics with the use of logarithmic amplification, and 10,000 events were analyzed for each antibody. The geometric mean fluorescent intensity (MFI) was used to quantitate the level of CD55 and CD59 expression. A cutoff for positive and negative cells was determined using the included calibrated bead with a lotspecific multiplier. Cells falling below this predetermined cutoff were considered abnormal. More than 3\% of abnormal cells are required for an abnormal test result for a particular RBC marker.

\section{Statistical Analyses}

Statistical data are presented as medians and 25 th and 75 th percentiles, as applicable. Associations between 2 or more independent groups were compared using either the $\chi^{2}$ test or Fisher's exact test, as appropriate. A value of $p \leq 0.05$ was considered statistically significant.

Data were analyzed using IBM SPSS, version 23 (IBM, Armonk, NY, USA). Quantitative variables were analyzed using descriptive statistics, including measures of location where means are reported with standard deviations and medians are reported with their interquartile ranges ( 25 th and 75 th percentiles) for paramet- 
Fig. 1. Comparative analysis of MFI of CD55 expression (a) and CD59 expression (b) from PRBC units and healthy controls.

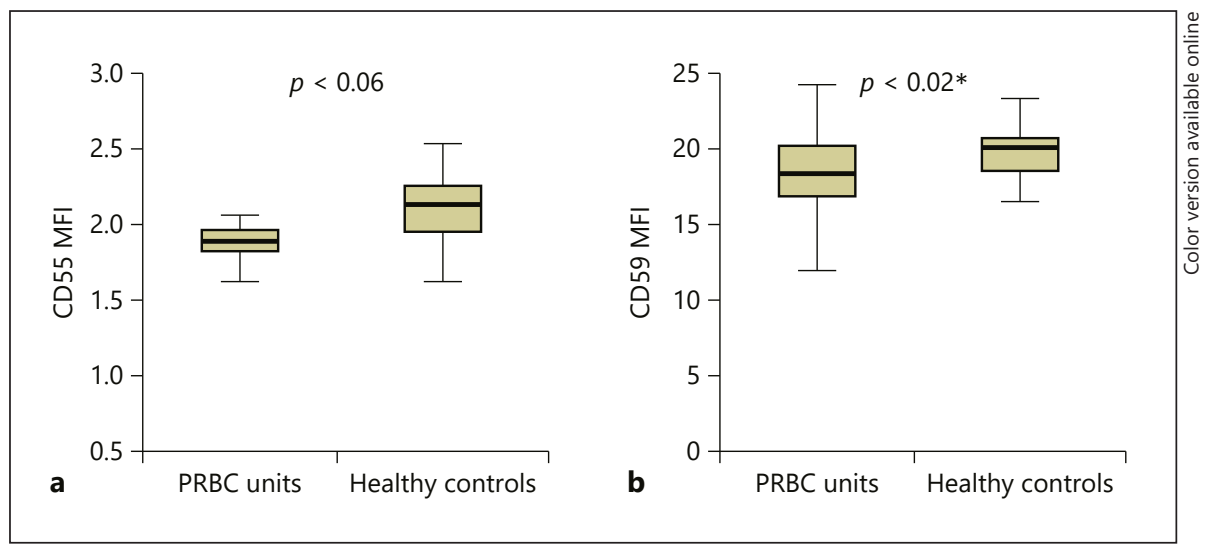

Table 1. Comparisons of CD55 and CD59 expression between all PRBC units with nonstored healthy control blood samples and between $\mathrm{PRBC}$ units of various age groups

\begin{tabular}{|c|c|c|c|c|c|c|c|}
\hline Parameters & $\begin{array}{l}\text { All PRBC } \\
\text { units }\end{array}$ & $\begin{array}{l}\text { Nonstored healthy } \\
\text { control samples }\end{array}$ & $p$ value & Group 1 & Group 2 & Group 3 & $p$ value \\
\hline$N$ & 92 & 56 & - & 28 & 35 & 29 & - \\
\hline CD59 deficiency, $n(\%)$ & $18(19.6)$ & $11(19.6)$ & 0.52 & - & - & - & - \\
\hline Median proportion of CD55-deficient RBCs, $\%$ & 0.1 & 0.1 & 0.76 & 0.1 & 0.1 & 0.1 & 0.09 \\
\hline $25-75 \%$ & $0.8-0.2$ & $0.04-0.2$ & & $0.07-0.12$ & $0.08-0.33$ & $0.06-0.19$ & \\
\hline MFI of CD55 expression (mean \pm SD) & $1.9(0.20)$ & $2.1(0.27)$ & 0.06 & $2.0(2.0)$ & $1.9(2.1)$ & $1.9(2.1)$ & 0.11 \\
\hline MFI of CD59 expression (mean \pm SD) & $17.8(3.8)$ & $20.2(3.5)$ & 0.02 & $20.5(2.9)$ & 17.1(4.9) & $16.5(2.2)$ & $<0.01$ \\
\hline
\end{tabular}

Significant differences are italicized.

ric and nonparametric variables, respectively. Parametric and nonparametric measures of comparison were used for symmetrical and skewed variables, respectively. A value of $p<0.05$ (two tailed) was considered statistically significant.

\section{Results}

\section{Data of Tested Blood Units}

Blood samples from segments of 92 PRBC units were collected. The solution added to all units was SAGM. The number in each group was 28 units in group 1, 35 units in group 2 , and 29 units in group 3 . ABO type distribution did not differ between the storage groups.

\section{Analysis of CD55 and CD59 on RBCs from PRBC \\ Units and Healthy Controls}

CD55 and CD59 deficiency states (CD55- and CD59deficient RBCs $>3 \%$ ) in PRBC units did not significantly differ when compared with healthy controls (Table 1). The proportions of CD55-and CD59-deficient RBCs from PRBC units did not significantly differ when compared with those from healthy controls ( 0.1 vs. 0.1 and 1.4 vs. 1.2, respectively) (Table 1). The MFI of CD59 expression on RBCs from PRBC units was significantly reduced compared to the expression on RBCs from healthy controls (17.8 vs. 20.2 , respectively); however, there were no significant differences in the MFI of CD55 expression on RBCs from both cohorts (1.9 vs. 2.1, respectively) (Table 1; Fig.1). A representative dot plot showing RBC distribution by forward scatter and side scatter, and histograms showing CD59 FITC MFI expression versus counted cells from RBC gate is shown in (Fig. 2).

\section{Analysis of CD55 and CD59 on RBCs from PRBC}

Units in Different Storage Age Groups

The proportions of CD55- and CD59-deficient RBCs from PRBC units did not significantly differ among the 3 


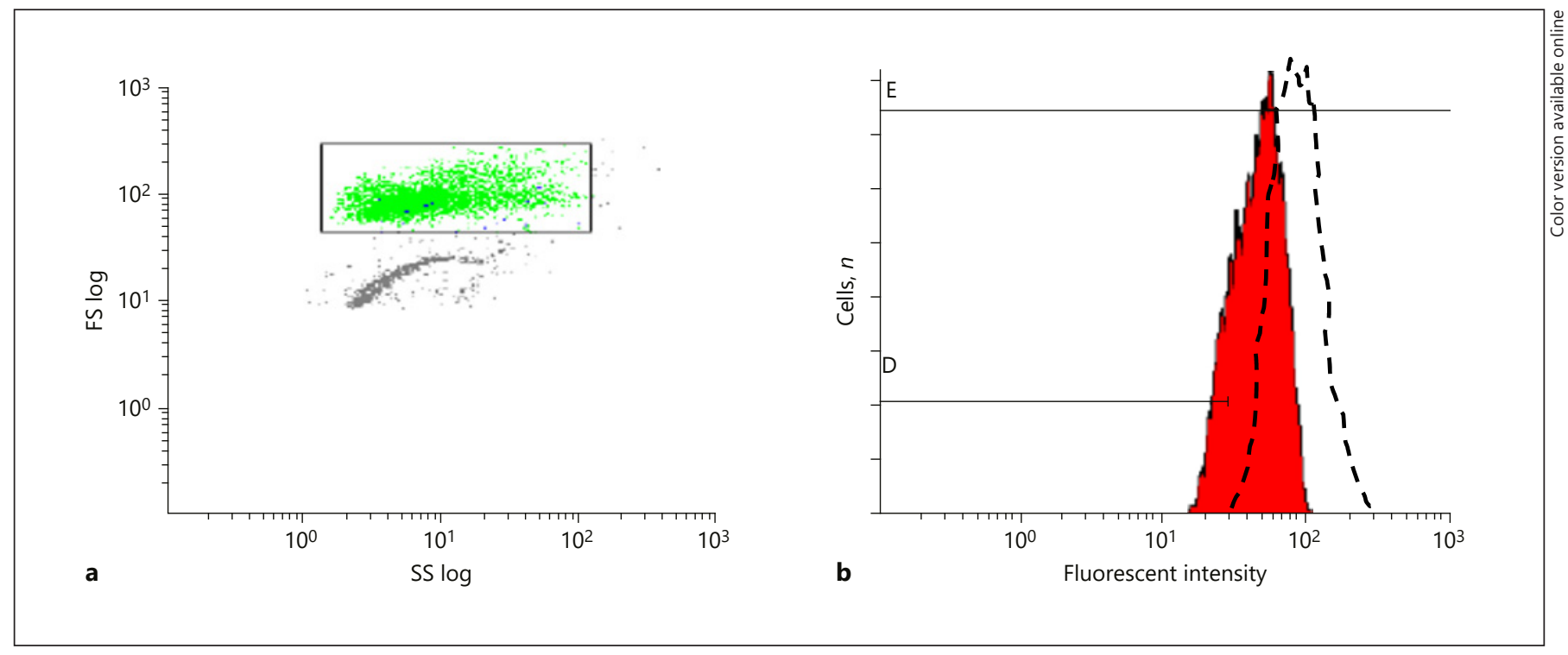

Fig. 2. Representative dot plots from a PRBC unit from group 3 showing RBC distribution and gating by forward (FS) and side scatter (SS) (a), and representative histogram of altered expression of CD59 in stored RBCs compared to healthy controls (dashed histogram) (b).

Fig. 3. Comparative analysis of MFI of CD59 expression among PRBC unit age groups. The dashed line indicates the cutoff value for CD59 negativity.

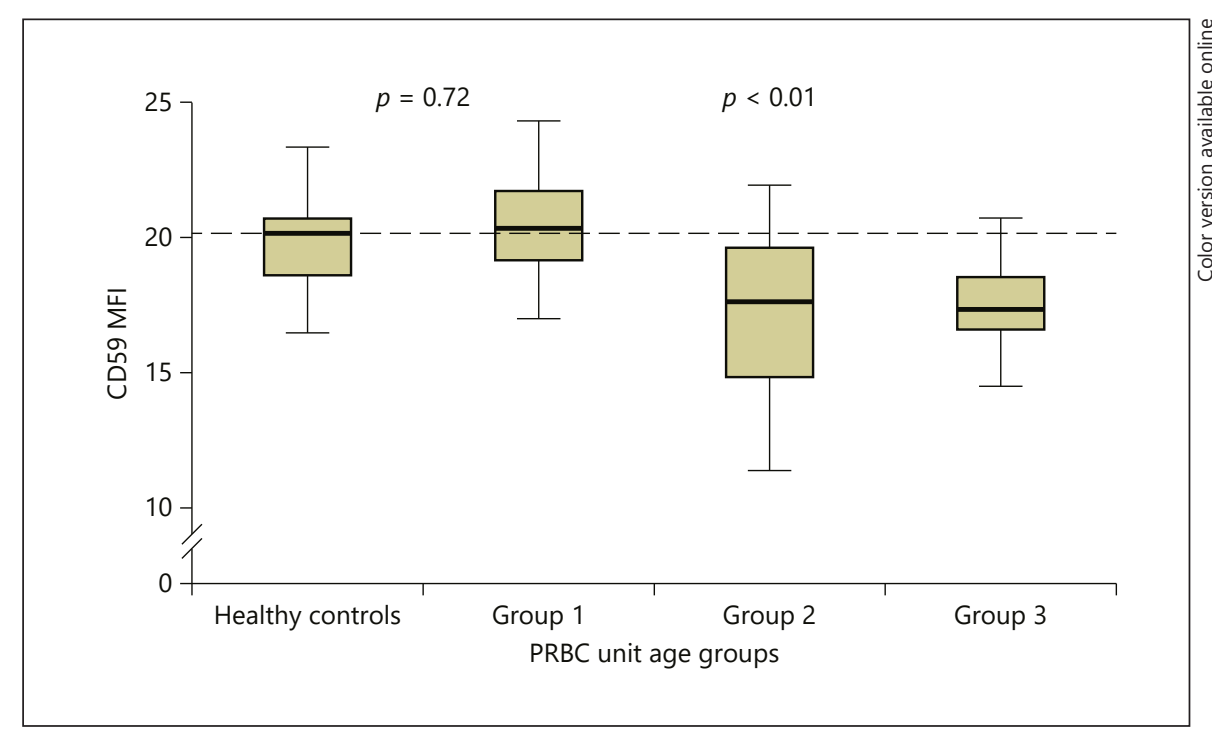

\section{Discussion}

Time-dependent changes, also known as storage lesion, reflect the deterioration in RBCs with storage. Many of the reported changes affect function and viability; however, information on structural time-dependent changes affecting $\mathrm{RBC}$ membrane proteins in stored blood is limited, and focused on vesiculation and the formation of microvesicles [14-16]. This study was aimed at examining the pattern of 
CD55 and CD59 expression on stored RBCs in PRBC units. As expected, the level of CD59 in stored RBCs in PRBC units was lower than that in fresh samples from healthy controls; however, although there was no statistically significant difference in the level of CD59 expression between stored RBCs and those from healthy controls, the biological significance of this difference needs to be further investigated. Interestingly, the MFI of CD55 expression on RBCs from stored blood did not significantly differ from that on RBCs from healthy controls. Unlike circulating blood, $\mathrm{RBCs}$ are stored at $2-6^{\circ} \mathrm{C}$ and diluted in a nonphysiological buffering system, which changes over time with respect to $\mathrm{pH}$ and ionic strength; this leads to the accumulation of other bioactive substances such as histamine, lipids, cytokines, and fragments of cellular membranes [1719]. These factors might play a role in the changes in CD55 and CD59 expression observed in our study. Unexpectedly, however, our data showed a trend to a gradual decline in CD59 expression, but not CD55 expression, on RBCs from PRBC units during the storage period of 42 days, as evidenced by the statistically significant reduction in MFI of CD59 compared to CD55.

Previous studies have shown conflicting findings. Our study did not reproduce the findings of Long et al. [13], who showed that both CD55 and CD59 molecules are lost during $\mathrm{RBC}$ storage, although they were also able to demonstrate a similar preferential decline in the level of CD59 expression compared to CD55 expression. Furthermore, in contrast to our study, Kamhieh-Milz et al. [20] and Freitas Leal et al. [21] showed significant loss of CD55, but not CD59, expression in stored RBCs. The discrepancy in the reported results could be attributed to several factors, including the use of different additive storage solutions, the use of different methodologies, and differences in the number of samples studied and their leukodepletion status, and the gating strategy used for flow-cytometric RBC analysis. In view of the fact that our results on the loss of CD55 are almost significant, it is likely to be so in a more powerful study.

It has been known that the attachment of the GPI-anchored proteins, CD55 and CD59, to plasma membranes via their inositol-phospholipids renders them potentially more displaceable from cell surfaces than conventionally anchored proteins. They are susceptible to cleavage and release by phosphatidyl inositol-specific phospholipase C, an enzyme that is present in mammalian tissues. CD55 and CD59 molecules with GPI anchors containing unacetylated inositol are preferentially lost [22]. If the GPI anchor structure of CD59 on stored RBCs does not differ from that of CD55, a defect in a GPI anchor assembly should affect the expression of both proteins in an equiva- lent manner. Bütikofer et al. [23] showed that GPI-anchored proteins are redistributed on the surface of human RBCs and preferentially depleted during vesiculation, which is one of the structural changes that occur in storage lesions. Whether the nonphysiological medium of the aging PRBC unit preferentially causes the displacement of an alternatively anchored CD59 (non-GPI-anchored CD59 molecule) form, rendering it more displaceable from the RBC surface, is a question that needs to be investigated in future studies. Interestingly, we have previously shown a similar expression pattern on RBCs from patients with sickle cell disease, in whom CD59 is preferentially lost compared to RBCs from healthy control subjects [24].

One limitation of this study might be the use of the REDQUANT CD55/CD59 kit. It is an indirect method, and thus the addition of the primary antibody is followed by a polyclonal anti-mouse IgG-FITC antibody. Therefore, conformational changes in the cell surface could render certain antigen sites hidden from the primary antibody or could mean that differing amounts of secondary antibody could bind. This means that the "loss" of antigens could be explained by crenation of the cells on storage. Furthermore, it is likely that RBCs are agglutinated by FITC-conjugated antibodies as this lowers the electronegativity of RBCs that naturally keeps them apart, and, therefore, may prevent further access for the secondary antibody that is potentially a possible cause for the lower signal intensity. Furthermore, the gating strategy used in this study might have underestimated the level of CD55 and CD59 expression. Using an anti-glycophorin antibody in gating might improve RBC isolation for the analysis of CD55 and CD59 expression, although leukodepletion of PRBCs is likely to minimize this effect. In addition, the vitality and properties of $\mathrm{RBC}$ stored in a tubing segment might be different from the RBC within the bag, which is a factor that might affect the results of this study.

\section{Conclusion}

The results of this study suggest that there is a preferential loss of CD59 expression, but not CD55 expression, on RBCs during blood storage. The significance of the altered CD59 expression during storage is unclear, and its physiological effect in vivo needs further investigation. In view of the results obtained in this study, further studies are needed to clarify their importance in the field of transfusion medicine, including in vitro studies correlating complement-mediated lysis with CD59 expression in stored blood. 


\section{Acknowledgments}

We thank Mrs. Sicy Easow for her tremendous efforts in performing the flow-cytometric analysis on the collected samples, and Mr. Generoso Gaytano for sample and data collection.

\section{Statement of Ethics}

The study protocol was approved by the Ethics Committees of Kuwait University's Health Sciences Centre and the Ministry of Health.

\section{Disclosure Statement}

The authors have no conflicts of interest to declare.

\section{Funding Sources}

Flow-cytometric analyses were performed in the Research Core Facility of the Health Sciences Center supported by Projects GM $01 / 01$ and GM 01/05. The work was supported by a grant from Research sectors, Kuwait University (MG01/14), which covered consumables, reagents, and temporary manpower.

\section{References}

1 American Association of Blood Banks (AABB). Standards for blood banks and transfusion services. 29th ed. Bethesda: AABB; 2014

2 Mollison PL, Young IM. In vivo survival in the human subject of transfused Erythrocytes after Storage in various preservative solutions. Q J Exp Physiol Cogn Med Sci. 1942; 31(4):359-92.

3 Mollison PL, Engelfriet CP, Contreras M. Blood transfusion in clinical medicine. 8 th ed. London: Blackwell; 1987.

4 Luten M, Roerdinkholder-Stoelwinder B, Schaap et al. Survival of red blood cells after transfusion: a comparison between red cells concentrates of different storage periods. Transfusion. 2008 Jul;48(7): 1478-85.

5 Hess JR. Red cell changes during storage. Transfus Apheresis Sci. 2010 Aug;43(1):51-9.

6 Blanchard D, Navenot JM, Petit-Le Roux Y, Willem C, Loirat MJ. Flow cytometry and immunoblotting analysis of monoclonal antibodies directed to complement regulatory proteins. Transfus Clin Biol. 1997;4(1):131-4.

7 Frank MM. Complement in the pathophysiology of human disease. N Engl J Med. 1987 Jun;316(24):1525-30.

8 Lublin DM, Atkinson JP. Decay-accelerating factor: biochemistry, molecular biology, and function. Annu Rev Immunol. 1989;7(1):3558.

9 Walport MJ. Complement. First of two parts. N Engl J Med. 2001 Apr;344(14):1058-66.
10 Nicholson-Weller A, March JP, Rosenfeld SI, Austen KF. Affected erythrocytes of patients with paroxysmal nocturnal hemoglobinuria are deficient in the complement regulatory protein, decay accelerating factor. Proc Natl Acad Sci USA. 1983 Aug;80(16):5066-70.

11 Holguin MH, Fredrick LR, Bernshaw NJ, Wilcox LA, Parker CJ. Isolation and characterization of a membrane protein from normal human erythrocytes that inhibits reactive lysis of the erythrocytes of paroxysmal nocturnal hemoglobinuria. J Clin Invest. 1989 Jul;84(1):7-17.

12 Telen MJ, Green AM. The Inab phenotype: characterization of the membrane protein and complement regulatory defect. blood, 1989 Jul;74(1):437-41.

13 Long KE, Yomtovian R, Kida M, Knez JJ, Medof ME. Time-dependent loss of surface complement regulatory activity during storage of donor blood. Transfusion. 1993 Apr; 33(4):294-300.

14 Almac E, Ince C. The impact of storage on red cell function in blood transfusion. Best Pract Res Clin Anaesthesiol. 2007 Jun;21(2):195-208.

15 Koch CG, Figueroa PI, Li L, Sabik JF 3rd, Mihaljevic T, Blackstone EH. Red blood cell storage: how long is too long? Ann Thorac Surg. 2013 Nov;96(5):1894-9.

16 Willekens FL, Werre JM, Groenen-Döpp YA, Roerdinkholder-Stoelwinder B, de Pauw B, Bosman GJ. Erythrocyte vesiculation: a selfprotective mechanism? Br J Haematol. 2008 May;141(4):549-56.

17 Gevi F, D’Alessandro A, Rinalducci S, Zolla L. Alterations of red blood cell metabolome during cold liquid storage of erythrocyte concentrates in CPD-SAGM. J Proteomics. 2012 Dec;76(Spec No):168-80.
18 Fransen E, Maessen J, Dentener M, Senden N, Buurman W. Impact of blood transfusions on inflammatory mediator release in patients undergoing cardiac surgery. Chest. 1999 Nov; 116(5):1233-9.

19 Smith KJ, Sierra ER, Nelson EJ. Histamine, IL$1 \mathrm{~B}$, and IL- 8 increase in Packed RBCs Stored for 42 days but not in RBCs Leukodepleted pre-storage. Transfusion. 1993;33[s]:53S.

20 Kamhieh-Milz J, Bartl B, Sterzer V, KamhiehMilz S, Salama A. Storage of RBCs results in an increased susceptibility for complementmediated degradation. Transfus Med. 2014 Dec;24(6):392-9.

21 Freitas Leal JK, Adjobo-Hermans MJ, Brock R, Bosman GJ. Acetylcholinesterase provides new insights into red blood cell ageing in vivo and in vitro. Blood Transfus. 2017 May;15(3):232-8.

22 Stieger S, Diem S, Jakob A, Brodbeck U. Enzymatic properties of phosphatidylinositol-glycan-specific phospholipase $\mathrm{C}$ from rat liver and phosphatidylinositol-glycan-specific phospholipase D from rat serum. Eur J Biochem. 1991 Apr;197(1):67-73.

23 Bütikofer P, Kuypers FA, Xu CM, Chiu DT, Lubin B. Enrichment of two glycosyl-phosphatidylinositol-anchored proteins, acetylcholinesterase and decay accelerating factor, in vesicles released from human red blood cells. Blood. 1989 Oct;74(5):1481-5.

24 Al-Faris L, Al-Rukhayes M, Al-Humood S. Expression pattern of CD55 and CD59 on red blood cells in sickle cell disease. Hematology. 2017 Mar;22(2):105-13. 